\title{
Kim-Type Critical State Models and Critical Currents of Thallium Based Superconductors
}

\author{
W.M. Woch ${ }^{a}$, R. ZAlecki ${ }^{a}$, A. KolodzIEJCZYK ${ }^{a}$, O. $\operatorname{HeimL}^{b}$, \\ H. SUDRA ${ }^{b}$ AND G. GRITZNER ${ }^{b}$ \\ ${ }^{a}$ Faculty of Physics and Applied Computer Science \\ AGH University of Science and Technology \\ al. Mickiewicza 30, PL-30-059, Kraków, Poland \\ ${ }^{b}$ Institute for Chemical Technology of Inorganic Materials \\ Johannes Kepler University, A-4040 Linz, Austria
}

The two extended formulae in the power form of the Kim critical state model were used to fit the critical currents versus the dc applied magnetic field. Two samples of thallium based superconductors: the $\left(\mathrm{Tl}_{0.6} \mathrm{~Pb}_{0.24} \mathrm{Bi}_{0.16}\right)\left(\mathrm{Sr}_{0.9} \mathrm{Ba}_{0.1}\right)_{2} \mathrm{Ca}_{2} \mathrm{Cu}_{3} \mathrm{O}_{y}$ film on single-crystalline lanthanum aluminate and the bulk $\left(\mathrm{Tl}_{0.5} \mathrm{~Pb}_{0.5}\right) \mathrm{Sr}_{2}\left(\mathrm{Ca}_{1.8} \mathrm{Gd}_{0.2}\right) \mathrm{Cu}_{2} \mathrm{O}_{y}$ were chosen to test the models. The formulae were compared to the percolation model described by the exponential expression. The first model fits the experimental data better for the thallium based film whereas the second approach is better for the thallium based bulk sample.

PACS numbers: 74.72.Jt, 74.25.Sv, 74.25.Ha

\section{Introduction}

The effect of the applied magnetic field on the critical current in high temperature superconductors (HTS) is strongly correlated with the vortex structure and with the vortex dynamics. The magnetic field dependence of the critical current is important especially for HTS because of their mixed inter- and intra-grain properties.

The magnetic field dependence of the critical current was firstly introduced by Kim et al. [1, 2] for hard superconductors. They assumed that the internal field is the algebraic sum of the applied magnetic field and the field produced by the current induced in the sample. They also assumed that the reverse of the critical current is proportional to the power series expansion

$$
\frac{a}{J}=H_{0}+H+a_{1} H^{2}+a_{2} H^{3}+\ldots,
$$


where $a$ and $H_{0}$ are material constants which can be determined from experiment and $H$ is the applied field. If the coefficients $a_{1}, a_{2}, \ldots$ are sufficiently small, Eq. (1) reduces to

$$
\frac{a}{J}=H_{0}+H
$$

This is the central result of the Kim critical state model that describes the critical current versus the applied magnetic field of the hard superconductors.

Equation (2) does not fit the magnetic dependences of the critical current of the HTS. Therefore, the extended models should be developed. The first model assumes that the $a / J$ is proportional to $H^{n}$, so Eq. (2) can be rewritten in the form

$$
\frac{a}{J} \propto H_{0}+H^{n}
$$

Taking it into account the field dependence of the critical current could be given in the following form [3]:

$$
J_{\mathrm{c}}=J_{\mathrm{c} 0}\left[1+\left(\frac{H}{H_{\mathrm{K} 1}}\right)^{n}\right]^{-1} .
$$

In this model the linear part of the magnetic field was neglected and one can expect that $n$ is not too large and usually less than two. This dependence will be discussed in this work.

The second model that is introduced in this paper assumes the following expansion:

$$
\frac{a}{J} \propto H_{0}+c_{1} H+c_{2} H^{2}
$$

where $c_{1}$ and $c_{2}$ are coefficients.

The simple calculation gives the formula for the field dependence of the critical current for this Kim extended critical state model as follows:

$$
J_{\mathrm{c}}=J_{\mathrm{c} 0}\left[1+\left(\frac{H}{H_{\mathrm{K} 2}}\right)\right]^{-n} .
$$

The $J_{\mathrm{c} 0}$ is the critical current at the zero magnetic field and the parameters $H_{\mathrm{K} 1}$ and $H_{\mathrm{K} 2}$ are material constants.

For $n=1$ the critical state models expressed by Eq. (4) and Eq. (6) give the original Kim model (2).

In this paper the Kim critical state model and its extensions (4) and (6) will be compared with the percolation critical state model. This model assumes a weak link network of the Josephson coupled grains with a critical current that has two components: the field-independent component and a field-dependent component that decays exponentially with the applied magnetic field [4]:

$$
J_{\mathrm{c}}=J_{\mathrm{c}}\left[\exp \left(-\frac{H}{H_{\mathrm{P}}}\right)+\alpha\right]
$$

where $J_{\mathrm{c}}(1+\alpha)$ is the critical current at the zero applied magnetic field and $H_{\mathrm{P}}$ is the material constant. 
The comparison will be done for the two samples: the first sample is $c$-axis oriented, $a b$ aligned $\left(\mathrm{Tl}_{0.6} \mathrm{~Pb}_{0.24} \mathrm{Bi}_{0.16}\right)\left(\mathrm{Ba}_{0.1} \mathrm{Sr}_{0.9}\right)_{2} \mathrm{Ca}_{2} \mathrm{Cu}_{3} \mathrm{O}_{y}$ film on (100) single-crystalline lanthanum aluminate substrate (Tl-1223 film) with the critical temperature of $T_{\mathrm{c}, 50 \%}=111.7 \mathrm{~K}$ and the width of the transitions $\Delta T=T_{90 \%}-$ $T_{10 \%}=3.0 \mathrm{~K}[5]$ and the second is the bulk $\left(\mathrm{Tl}_{0.5} \mathrm{~Pb}_{0.5}\right) \mathrm{Sr}_{2}\left(\mathrm{Ca}_{1.8} \mathrm{Gd}_{0.2}\right) \mathrm{Cu}_{2} \mathrm{O}_{y}$ (Tl-1212 bulk) superconductor with the critical temperature $T_{\mathrm{c}, 50 \%}=105.3 \mathrm{~K}$ and the width of the transitions $\Delta T=T_{90 \%}-T_{10 \%}=3.6 \mathrm{~K}[6]$.

\section{Results and their analysis}

\section{1. $\left(\mathrm{Tl}_{0.6} \mathrm{~Pb}_{0.24} \mathrm{Bi}_{0.16}\right)\left(\mathrm{Ba}_{0.1} \mathrm{Sr}_{0.9}\right)_{2} \mathrm{Ca}_{2} \mathrm{Cu}_{3} \mathrm{O}_{y}$ film}

The magnetic field dependence of the critical current of the Tl-1223 film shows the typical behavior: first the critical current drops rapidly in the low field range and next the current goes to be field independent. This effect is noticeable especially for the $H \| c$ direction. The data fitted by the extended model described by Eq. (4) are shown in Fig. $1 \mathrm{a}$ and b. There are two fitting parameters: $J_{\mathrm{c} 0}$ and
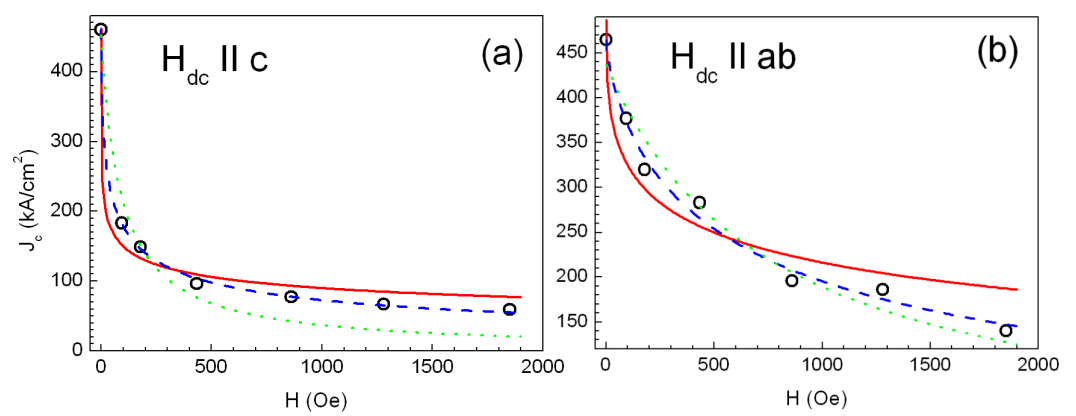

Fig. 1. Critical current vs. the applied magnetic field of the Tl-1223 film (open circles) fitted by the extended model expressed by Eq. (4) (solid line for $n=0.3$, dashed one for $n=0.53$, dotted one for $n=1.0(H \| c)$ and solid line for $n=0.4$, dashed one for $n=0.73$, dotted one for $n=1.0(H \| a b))$.

$H_{\mathrm{K} 1}$ and exponent $n$ which has been stated to get the best fit. These fitting parameters are collected in Table I. The best fit was reached for the exponent $n=0.53$ for $H \| c$ and $n=0.73$ for $H \| a b$ direction (the dashed lines in Fig. 1a and b). The lower as well as the higher exponents $n$ than $n=0.53$ for $H \| c$ and $n=0.73$ for $H \| a b$ give worse shaping to the experimental data. The data fitted by the second Kim extended model described by Eq. (6) are shown in Fig. 2a and b. There are also two fitting parameters: $J_{\mathrm{c} 0}$ and $H_{\mathrm{K} 2}$ and exponent $n$ that has been stated to get the best fit. These fitting parameters are collected in Table II. The best fit was reached for the exponent $n=0.4$ for $H \| c$ and $n=0.45$ for $H \| a b$ direction (the dashed lines in Fig. 2a and b). The lower as well as the higher exponents $n$ than $n=0.4$ for $H \| c$ and $n=0.45$ for $H \| a b$ give worse shaping to the experimental data likely as in the previous case. It should be emphasized that 

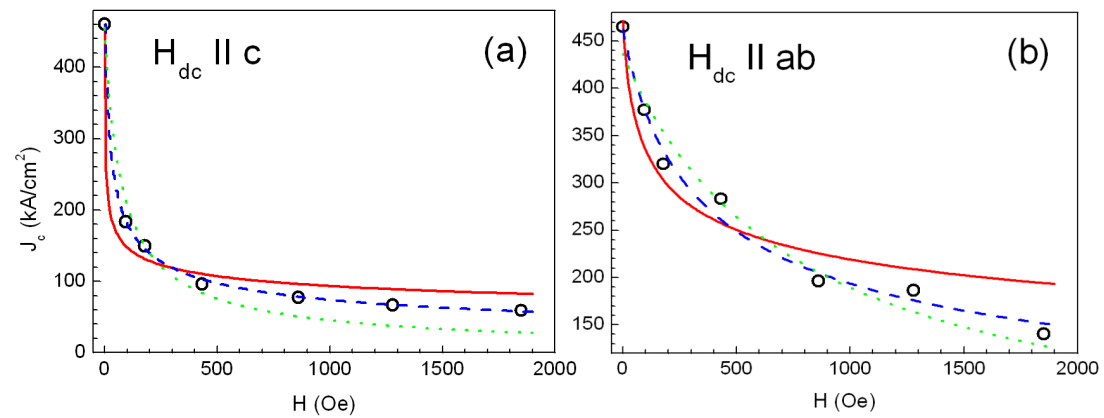

Fig. 2. Critical current vs. the applied magnetic field of the Tl-1223 film (open circles) fitted by the extended model expressed by Eq. (6) (solid line for $n=0.2$, dashed one for $n=0.4$, dotted one for $n=0.8(H \| c)$ and solid line for $n=0.2$, dashed one for $n=0.45$, dotted one for $n=1.0(H \| a b))$.

TABLE I

Fitting parameters $J_{\mathrm{c} 0}$ and $H_{\mathrm{K} 1}$ for the magnetic field dependence of the critical current density according to Eq. (4).

\begin{tabular}{|c|c|c|c|}
\hline & & $J_{\mathrm{c} 0}\left[\mathrm{kA} / \mathrm{cm}^{2}\right]$ & $H_{\mathrm{K} 1}[\mathrm{Oe}]$ \\
\hline \multirow{3}{*}{$H \| c$} & $n=0.3$ & $462 \pm 21$ & $9 \pm 4$ \\
\hline & $n=0.53$ (best fit) & $460 \pm 4$ & $42 \pm 2$ \\
\hline & $n=1.0$ & $452 \pm 34$ & $89 \pm 20$ \\
\hline \multirow{3}{*}{$H \| a b$} & $n=0.4$ & $486 \pm 37$ & $570 \pm 300$ \\
\hline & $n=0.73$ (best fit) & $464 \pm 11$ & $645 \pm 67$ \\
\hline & $n=1.0$ & $437 \pm 18$ & $764 \pm 118$ \\
\hline
\end{tabular}

TABLE II

Fitting parameters $J_{\mathrm{c} 0}$ and $H_{\mathrm{K} 2}$ for the magnetic field dependence of the critical current density according to Eq. (6).

\begin{tabular}{c|c|c|c}
\hline \hline \multicolumn{2}{l|}{} & $J_{\mathrm{c} 0}\left[\mathrm{kA} / \mathrm{cm}^{2}\right]$ & $H_{\mathrm{K} 2}[\mathrm{Oe}]$ \\
\hline \multirow{3}{*}{$H \| c$} & $n=0.2$ & $460 \pm 25$ & $0.34 \pm 0.18$ \\
& $n=0.4$ (best fit) & $460 \pm 4$ & $10.4 \pm 0.4$ \\
& $n=0.8$ & $455 \pm 27$ & $59 \pm 12$ \\
\hline \multirow{3}{*}{$H \| a b$} & $n=0.2$ & $471 \pm 37$ & $22 \pm 12$ \\
& $n=0.45$ (best fit) & $462 \pm 13$ & $169 \pm 21$ \\
& $n=1.0$ & $437 \pm 18$ & $765 \pm 118$
\end{tabular}

the exponents $n$ are very similar for both $H \| c$ and $H \| a b$ directions and in this sense one can say that it is more universal. According to the chi-square test the best fitting of Eq. (6) is better than of Eq. (4). To test the results of these fitting 
TABLE III

Fitting parameters $J_{\mathrm{c}}$ and $H_{\mathrm{P}}$ for the magnetic field dependence of the critical current density according to Eq. (7).

\begin{tabular}{c|c|c|c}
\hline \hline \multicolumn{2}{l|}{} & $J_{\mathrm{c}}\left[\mathrm{kA} / \mathrm{cm}^{2}\right]$ & $H_{\mathrm{P}}[\mathrm{Oe}]$ \\
\hline \multirow{3}{*}{$H \| c$} & $\alpha=0.0$ & $439 \pm 65$ & $152 \pm 51$ \\
& $\alpha=0.2$ (best fit) & $381 \pm 15$ & $85 \pm 10$ \\
& $\alpha=0.4$ & $249 \pm 27$ & $66 \pm 30$ \\
\hline \multirow{3}{*}{$H \| a b$} & $\alpha=0.0$ & $410 \pm 24$ & $1407 \pm 243$ \\
& $\alpha=0.53$ (best fit) & $291 \pm 12$ & $434 \pm 83$ \\
& $\alpha=1.0$ & $210 \pm 18$ & $310 \pm 176$
\end{tabular}
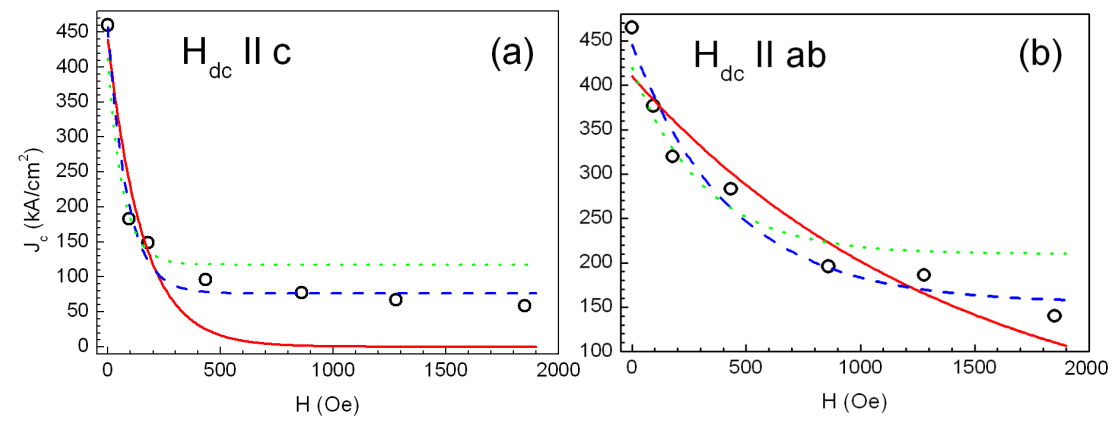

Fig. 3. Critical current vs. the applied magnetic field of the Tl-1223 film (open circles) fitted by the percolation critical state model expressed by Eq. (7) (solid line for $\alpha=0.0$, dashed one for $\alpha=0.2$, dotted one for $\alpha=0.4(H \| c)$ and solid line for $\alpha=0.0$, dashed one for $\alpha=0.53$, dotted one for $\alpha=1.0(H \| a b))$.

procedures one can compare the fitting parameters $J_{\mathrm{c} 0}$ with the experimental critical current measured by transport method: $J_{\mathrm{c} 0}=460 \mathrm{kA} / \mathrm{cm}^{2}$. The best fit of the critical state models represented by Eq. (4) as well as by Eq. (6) gives the fitting parameters $J_{\mathrm{c} 0}$ identical or very close to the measured critical current at the zero magnetic field.

The extensions of the Kim critical state model have been compared with the percolation critical state model. The data fitted by the percolation model described by Eq. (7) are shown in Fig. 3a and b. There are also two fitting parameters: $J_{\mathrm{c}}$ and $H_{\mathrm{P}}$ and constant $\alpha$ which has been stated to get the best fit. These fitting parameters are collected in Table III. The best fit was reached for the constant $\alpha=0.2$ for $H \| c$ and $\alpha=0.53$ for $H \| a b$ direction (the dashed lines in Fig. 3a and b). The lower as well as the higher constants $\alpha$ than $\alpha=0.2$ for $H \| c$ and $\alpha=0.53$ for $H \| a b$ give worse shaping to the experimental data. The fitting lines that represent Eqs. (4) and (6) are almost the same and they fit the data much better than the solid line which presents the fitting to Eq. (7). 
Summarizing we shall notice that for the Tl-1223 film the exponent $n$ in Eq. (4) as well as in Eq. (6) is lower than one and its value is close to 0.5 for the best fits. The constants of a material $H_{\mathrm{K} 1}$ and $H_{\mathrm{K} 2}$ are comparable for the both models but the $H_{\mathrm{K} 1}$ should be powered to the exponent $n$.

$$
\text { 2.2. Bulk ( } \left.\mathrm{Tl}_{0.5} \mathrm{~Pb}_{0.5}\right) \mathrm{Sr}_{2}\left(\mathrm{Ca}_{1.8} \mathrm{Gd}_{0.2}\right) \mathrm{Cu}_{2} \mathrm{O}_{y}
$$

The data fitted by the model described by Eq. (4) are shown in Fig. 4. There are also two fitting parameters: $J_{\mathrm{c} 0}$ and $H_{\mathrm{K} 1}$ and exponent $n$ that has been stated to get the best fit. These fitting parameters are collected in Table IV. The best fit was reached for the exponent $n=1.6$ (the dotted line in Fig. 4). The lower as well as the higher exponents $n$ than $n=1.6$ give worse shaping to the experimental data. The experimental points fitted by the model described

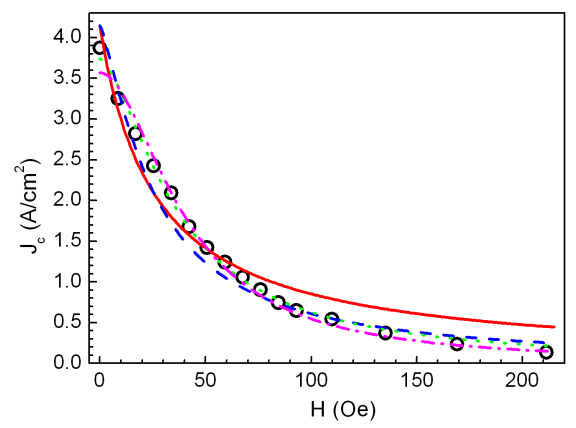

Fig. 4. Critical current vs. the applied magnetic field of the bulk Tl-1212 sample (open circles) fitted by the extended model expressed by Eq. (4) (solid line for $n=1.0$, dashed one for $n=1.3$, dotted one for $n=1.6$, dashed-dotted one for $n=1.9$ ).
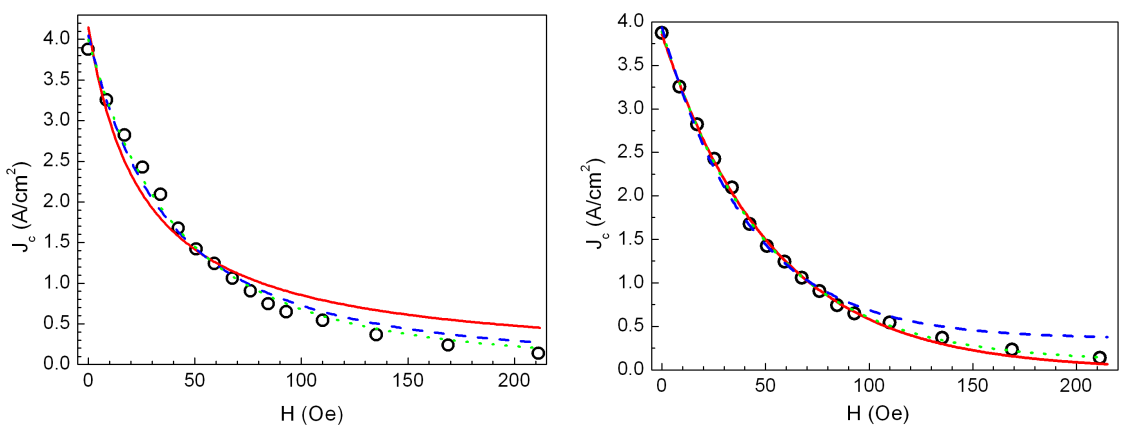

Fig. 5. Critical current vs. the applied magnetic field of the bulk Tl-1212 sample (open circles) fitted by the extended model expressed by Eq. (6) (solid line for $n=1$, dashed one for $n=2$, dotted one for $n=3$ ).

Fig. 6. Critical current vs. the applied magnetic field of the bulk Tl-1212 sample (open circles) fitted by the percolation critical state model expressed by Eq. (7) (solid line for $\alpha=0.000$, dashed one for $\alpha=0.025$, dotted one for $\alpha=0.100$ ). 
TABLE IV

Fitting parameters $J_{\mathrm{c} 0}$ and $H_{\mathrm{K} 1}$ for the magnetic field dependence of the critical current density according to Eq. (4).

\begin{tabular}{c|c|c}
\hline \hline & $J_{\mathrm{c} 0}\left[\mathrm{~A} / \mathrm{cm}^{2}\right]$ & $H_{\mathrm{K} 1}[\mathrm{Oe}]$ \\
\hline$n=1.0$ & $4.15 \pm 0.22$ & $26 \pm 3$ \\
$n=1.3$ & $4.15 \pm 0.17$ & $26 \pm 2$ \\
$n=1.6$ (best fit) & $3.74 \pm 0.06$ & $37 \pm 1$ \\
$n=1.9$ & $3.57 \pm 0.08$ & $40.7 \pm 1.5$
\end{tabular}

TABLE V

Fitting parameters $J_{\mathrm{c} 0}$ and $H_{\mathrm{K} 2}$ for the magnetic field dependence of the critical current density according to Eq. (6).

\begin{tabular}{l|c|c}
\hline \hline & $J_{\mathrm{c} 0}\left[\mathrm{~A} / \mathrm{cm}^{2}\right]$ & $H_{\mathrm{K} 2}[\mathrm{Oe}]$ \\
\hline$n=1$ & $4.20 \pm 0.22$ & $26 \pm 3$ \\
$n=2$ & $4.05 \pm 0.11$ & $74 \pm 4$ \\
$n=3$ & $4.00 \pm 0.07$ & $124 \pm 4$
\end{tabular}

TABLE VI

Fitting parameters $J_{\mathrm{c}}$ and $H_{\mathrm{P}}$ for the magnetic field dependence of the critical current density according to Eq. (7)

\begin{tabular}{c|c|c}
\hline \hline & $J_{\mathrm{c}}\left[\mathrm{kA} / \mathrm{cm}^{2}\right]$ & $H_{\mathrm{P}}[\mathrm{Oe}]$ \\
\hline$\alpha=0.000$ & $3.86 \pm 0.04$ & $52.9 \pm 0.9$ \\
$\alpha=0.025$ & $3.80 \pm 0.03$ & $49.8 \pm 1.1$ \\
$\alpha=0.100$ & $3.58 \pm 0.08$ & $42 \pm 2$
\end{tabular}

by Eq. (6) are shown in Fig. 5. The fitting parameters are given in Table V. In this case the fitting procedure has not a minimum but chi-square test changes asymptotically if the parameter $n$ increases. For $n=3$ (the dotted line in Fig. 5) the chi-square test is almost the same as for $n=1.6$ in Eq. (4). The percolation critical state model described by Eq. (7) fits the experimental data better than the critical state model (see Fig. 6) described by Eqs. (4) and (6). The constant $\alpha$ is very close to zero for the good fitting (see the fitting parameters given in Table VI). As was mentioned above to test the results of these fitting procedures one can compare the fitting parameters $J_{\mathrm{c} 0}$ with the experimental critical current measured by transport method of this sample: $J_{\mathrm{c} 0}=3.88 \mathrm{~A} / \mathrm{cm}^{2}$. The best fit of 
the critical state models represented by Eq. (7) gives the fitting parameters $J_{\mathrm{c} 0}$ very close to the measured critical current at the zero magnetic field.

\section{Conclusions}

The results of this paper may be summarized as follows:

1. The model expressed by Eq. (2) does not describe our results within measured interval of the applied magnetic fields.

2. The extended critical state models expressed by Eq. (4) and introduced in this paper by Eq. (6) fit experimental data very well.

3. The percolation critical state model is an alternative approach to describe the critical state in HTS.

4. The exponent $n$ in the extended models (Eqs. (4) and (6)) is less than one for aligned Tl-1223 film. The model expressed by Eq. (6) fits the critical current versus the applied magnetic field in the best way.

5. The exponent $n$ in the extended models is higher than one for bulk Tl-1212 sample. The percolation critical state model expressed by Eq. (7) fits the critical current versus the applied magnetic field of that sample in the best way.

\section{Acknowledgments}

This project was supported by the Faculty of Physics and Applied Computer Science, AGH University of Science and Technology, Cracow, Poland and by the Austrian Science Foundation (Fonds zur Förderung der Wissenschaftlichen Forschung in Österreich, project number 17420-N07).

\section{References}

[1] Y.B. Kim, C.F. Hempstead, A.R. Strand, Phys. Rev. Lett. 9, 306 (1962).

[2] Y.B. Kim, C.F. Hempstead, A.R. Strand, Phys. Rev. 129, 528 (1963).

[3] M. Ciszek, B.A. Głowacki, S.P. Ashworth, A.M. Campbell, W.Y. Liang, R. Flukiger, R.E. Gladyshevskii, Physica C 260, 93 (1996).

[4] T.B. Doyle, R.A. Doyle, Phys. Rev. B 47, 8111 (1993).

[5] W.M. Woch, R. Zalecki, A. Kołodziejczyk, O. Heiml, G. Gritzner, Physica C 434, 17 (2006).

[6] W.M. Woch, R. Zalecki, A. Kołodziejczyk, H. Sudra, G. Gritzner, unpublished results. 